\title{
Localization Scheme of Assistive Tools for Print-Disabled People to Access STEM Contents
}

\author{
Katsuhito Yamaguchi ${ }^{1(\varpi)}$ and Masakazu Suzuki ${ }^{2}$ \\ 1 Junior College Funabashi Campus, Nihon University, \\ 7-24-1 Narashinodai, Funabashi, Chiba 274-8501, Japan \\ eugene@gaea.jcn.nihon-u.ac.jp \\ 2 Institute of Mathematics for Industry, Kyushu University, \\ 744, Motooka, Nishi-Ku, Fukuoka 819-0395, Japan \\ msuzuki@kyudai.jp
}

\begin{abstract}
To facilitate the spread of accessible e-books, especially books in STEM much more in developing countries, an efficient/ systematic scheme to localize producing/reading tools should be established. Here, multilingual support in our tool to produce accessible STEM contents and problems in localizing it are discussed. Our new localization scheme and the localization for Vietnamese as a model case are shown.
\end{abstract}

Keywords: DAISY $\cdot \mathrm{STEM} \cdot$ Localization $\cdot$ Print-disabilities Developing country

\section{Introduction}

It is said that $90 \%$ of visually disabled people live in the developing countries. Recently, accessible e-books are becoming gradually available even in those countries. For instance, "the Accessible Books Consortium (ABC)" provides training and technical assistance in developing countries such as India, Bangladesh, Nepal and Sri Lanka since 2015 in the production and distribution of books in accessible formats [1].

As is well known, in Western countries, "DAISY (Digital Accessible Information System)" [2] (or accessible EPUB3 that is essentially DAISY4) has already held the position of the international standard for accessible e-books. Many excellent tools to produce and to read DAISY/accessible EPUB3 books are available. Certainly, DAISY is also a key technology in the activities for developing countries mentioned above. However, to facilitate the spread of accessible books much more in such countries, even now, we have several problems. For instance, a text-to-speech (TTS) engine for their local language is usually not available or of low quality. It is rather hard to produce accessible books in text DAISY or multimedia DAISY (DAISY3 or accessible EPUB3) with TTS technology.

In those countries, it is particularly difficult for print-disabled people to obtain accessible e-books in STEM (science, technology, engineering and math).

(C) The Author(s) 2018

K. Miesenberger and G. Kouroupetroglou (Eds.): ICCHP 2018, LNCS 10896, pp. 606-610, 2018.

https://doi.org/10.1007/978-3-319-94277-3_94 
Recently, our group, NPO: "Science Accessibility Net (sAccessNet)" [3] has been working on developing tools to produce accessible STEM contents for developing countries by localizing our software. In this paper, the current situation of STEM accessibility is briefly reviewed. Next, multilingual support in our tool to produce/read multimedia-DAISY STEM books and problems in localizing such tool are discussed. Finally, our new localization scheme and a model case of the localization are shown.

\section{STEM Accessibility}

For the past more-than-20 years, various computerized approaches have been tried to improve STEM accessibility. In 1994, T. V. Raman reported on his "Audio System for Technical Readings" which could read out math documents in LaTeX format with DEC Talk synthesizer. It might be the very first trial in this field [5]. While LaTeX plays an important role in STEM accessibility even now, recently, MathML has become a key technology in assistive tools for printdisabled people to access STEM. For instance, DAISY has adopted MathML to represent math contents [2].

For about 20 years, sAccessNet and our research group, "Infty Project," [4] have been also developing assistive tools for print-disabled people to access STEM contents. "ChattyInfty" $[3,4]$ is an accessible math-document editor with a TTS voice. Using it, print-disabled people not only can read but also author a STEM document easily for themselves in an intuitive manner. It can help them with both accessing and doing math. It uses Microsoft Speech API, Ver.5 (SAPI5) as a TTS engine.

The current version, "ChattyInfty3" can be used as an authoring tool for accessible STEM books since the edited result can be saved as a multimedia DAISY/accessible EPUB3 book. Combining ChattyInfty3 with our OCR software for STEM documents "InftyReader" [3,4,6], users can convert a STEM document quite efficiently into multimedia DAISY/accessible EPUB3.

\section{Multilingual Support in ChattyInfty}

We have been releasing the Japanese and English versions of ChattyInfty since the very beginning of it. To release both the English and the Japanese versions efficiently/systematically, in ChattyInfty, not only tables to assign aloud reading math symbols/formulas but also captions in menu items and dialogs are all stored in an independent file on a main program. Thus, in principle, we could customize ChattyInfty3 for each local language. We have actually developed its French, Italian, Czech and Turkish (trial) versions during the last four years.

In 2016, a research group in India inquired of us if a Hindustani version of ChattyInfty could be developed. We thoroughly examined the possibility. Unfortunately, however, we concluded that it should not be so easy to realize 
since the character set in Hindustani was quite different from European languages/Japanese. To treat Hindustani, we must reform thoroughly the editor program of ChattyInfty, itself. Thus, this plan is suspended for the present.

\section{New Localization Scheme in ChattyInfty}

Through the experience of trying to develop the Hindustani version, we concluded that we should give a much better localization scheme for ChattyInfty3. As the first step to realize that, we have recently implemented the following new features in ChattyInfty3.

(1) Unicode can be used on its main window so that users can input a text in their local language if characters are included in Unicode.

(2) The definition file for reading aloud math symbols and formulas, "ReadSetting.txt," is also represented in Unicode so that users can prepare its local-language version.

(3) STEM terminology in menu items can be also replaced with local names. Ordinary menu items such as "File" might be OK even if they were represented in English. In developing countries, users are usually familiar with the menu items in English; however, names of technical symbols and math formulas such as "Square Root" should be represented in their local manner.

(4) Any SAPI5 voices can be selected for speech output.

As a model case of the localization, in cooperation with "Assistive Technology Developing Organization (ATDO)" [7], we have worked on developing the Vietnamese version in 2017 [8]. Vietnamese is represented in alphabet with various (different eight types) accent marks; those characters are all included in Unicode. Using an application named "Unikey," we can input Vietnamese easily with the ordinary keyboard. Vietnamese SAPI5 TTS engines are rather of low quality but available, anyhow. Thus, considering such situations, we have judged the localization should be possible.

In May 2017, ATDO, sAccessNet and a user group in Vietnam held meetings in Hanoi to discuss how we have taken responsibility for completing the mission. The ATDO and Vietnamese members were to undertake the following jobs.

(1) They would translate the English versions of the definition file, ReadSetting.txt and its manual into Vietnamese.

(2) They would list up menu items that should be represented in Vietnamese and give us their local names.

(3) They would also prepare sample STEM documents in Vietnamese to test how the software would work.

In addition to the list mentioned previously, we revised ChattyInfty so that Unikey could be used to input. We would incorporate the Vietnamese version of ReadSetting.txt and the indicated local names of the menu items into the software. 
Those jobs were completed by the mid October 2017, and we held workshops at the Hanoi School for the Blind in the end October 2017 to demonstrate how the Vietnamese version of ChattyInfty worked. The participants confirmed that they could write and read various math expressions with Vietnamese speech output. A blind high-school student who participated in the workshop said that he was so happy that he could write any math expressions freely.

However, we willingly admit that our scheme is not useful enough, yet. For the moment, we (the software developer) must execute a build process to incorporate ReadSetting.txt and the local names of the menu items in the software. Furthermore, we cannot check it for ourselves if a local TTS engine works properly. Each TTS engine has its own characteristics, and even if the same definition file for aloud reading were incorporated, actual speech output would be different from each other, depending on a used engine. Unexpected errors in reading could occur due to an inappropriate description in ReadSetting.txt. Thus, in cooperation with a local-user community, we must check speech output for various sample files and do necessary corrections. It requires us to exchange data with the local-user community over and over. It is obviously impossible for us to work on all requested localization for ourselves. Thus, we must give a much more efficient/systematic method to allow each local-user community to do most of necessary jobs just for themselves.

To achieve the objective, we are now developing a build tool for end users to allow themselves to perform the process to incorporate the ReadSetting.txt and the local names of the menu items in the software without any help of the developer. It will be included in the ChattyInfty3 package by the ICCHP2018.

\section{Conclusion}

When our localization scheme is completed, if the local version of ReadSetting.txt and the local names of the menu items were just prepared, users could customize ChattyInfty easily for themselves so that it could treat STEM contents in their own languages. We believe that our software could contribute to improve accessibility, especially STEM accessibility much more in developing countries.

\section{References}

1. ABC. http://www.accessiblebooksconsortium.org/portal/en/

2. The DAISY Consortium. http://www.daisy.org/

3. sAccessNet. http://www.sciaccess.net/en/

4. Infty Project. http://www.inftyproject.org/en/

5. Raman, T.V.: Audio system for technical readings. Dissertation, Cornell University (1994). Springer, Berlin (1998)

6. Suzuki, M., Yamaguchi, K.: Recognition of E-born PDF including mathematical formulas. In: Miesenberger, K., Bühler, C., Penaz, P. (eds.) ICCHP 2016. LNCS, vol. 9758, pp. 35-42. Springer, Cham (2016). https://doi.org/10.1007/978-3-31941264-1_5 
7. ATDO. http://atdo.website/english/

8. Yamaguchi, K., Kanahori, T., Suzuki, M., (Hamada) Makio, M.: Activities to provide accessible STEM E-books for the developing countries. In: The 33rd CSUN Assistive Technology Conference, EDU-058, San Diego (2018)

Open Access This chapter is licensed under the terms of the Creative Commons Attribution 4.0 International License (http://creativecommons.org/licenses/by/4.0/), which permits use, sharing, adaptation, distribution and reproduction in any medium or format, as long as you give appropriate credit to the original author(s) and the source, provide a link to the Creative Commons license and indicate if changes were made.

The images or other third party material in this chapter are included in the chapter's Creative Commons license, unless indicated otherwise in a credit line to the material. If material is not included in the chapter's Creative Commons license and your intended use is not permitted by statutory regulation or exceeds the permitted use, you will need to obtain permission directly from the copyright holder.

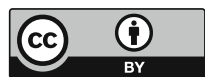

